\title{
Simple emergence structures from complex magnetic fields
}

\author{
M. J. Murray and A. W. Hood
}

\author{
School of Mathematics and Statistics, University of St. Andrews, North Haugh, St. Andrews, Fife KY16 9SS, UK \\ e-mail: michelle@mcs.st-and.ac.uk
}

Received 7 February 2007 / Accepted 17 April 2007

\begin{abstract}
Context. There has been suggestions that the initial subsurface magnetic field in flux emergence models is too organised. Thus, it may be that the results they yield are very specific and cannot be applied to the generic flux emergence process occurring on the Sun. Aims. We will demonstrate that the interaction of two flux tubes can increase the complexity of the solar interior's magnetic field. However, the subsequent emergence of this complex field results in the atmospheric flux having a simple and generic structural form. Methods. Using a numerical 3D MHD code, we consider the evolution of two flux tubes within a vertically stratified domain, representing the upper layers of the solar interior through to the corona. The flux tubes are initially situated in the solar interior, one tube lying directly above the other. By choosing the lower tube to be buoyant, whilst the upper tube is in mechanical equilibrium with its surroundings, we encourage the interaction of the two tubes within the solar interior. Thus, we will create a more complex magnetic field lying just below the photospheric surface than would be generated with a single flux tube model. By studying the subsequent emergence and structure of the resulting atmospheric field we will be able to identify if there are any significant changes due to the increased complexity of the field below the surface.

Results. The reconnection process modifies the topology of the subsurface magnetic field as indicated by the results of previous simulations. Since reconnection is occurring in a high plasma- $\beta$ region, where the sound speed is greater than the Alfvén speed, we observe no significant jets and also find no significant heating. When this reconnected field comes to the surface it is complex but the emergence occurs in much the same manner as for a single flux tube and, therefore, produces very little change in the atmospheric field. This strongly indicates that the results of previous emergence simulations are valid for both simple and complex subsurface fields.
\end{abstract}

Key words. magnetohydrodynamics (MHD) - Sun: magnetic fields - Sun: interior - Sun: atmosphere - methods: numerical

\section{Introduction}

The emergence of magnetic flux from the solar interior into the atmosphere of the Sun in evident on many scales. Millions of small scale magnetic fragments make up the magnetic carpet of the quiet sun. Through their emergence, submergence and interaction, the makeup of the carpet is constantly evolving. On larger scales, emergence is evident in the birth of new active regions. As large bundles of magnetic flux emerge from the solar interior, we observe the creation and growth of individual sunspot groups, where the spots mark the intersection of the magnetic field with the visible solar surface.

It is widely believed that the magnetic flux we see emerging through the solar surface in active regions originated deep inside the Sun through dynamo action. The dynamo process turns poloidal magnetic field into toroidal field via differential rotation. Combinations of rotational shear and turbulent motions in the convection zone fashion the magnetic field into flux bundles, or tubes, and, under buoyancy, these bundles rise towards the surface forming $\Omega$-shaped loops (Parker 1955). Upon reaching the surface, the flux can only emerge into the atmosphere if it undergoes a magnetic buoyancy instability (Matsumoto \& Shibata 1992; Kusano et al. 1998; Archontis et al. 2004).

The form of the field prior to emergence is, to a large extent, unknown. Using observational measurements, theoretical models for general and individual flux emergence events have been created (Glackin 1975; Parker 1979; Lites et al. 1995; Strous \& Zwaan 1999). From these models, predictions have been made about the subsurface magnetic field and have provided input for many of the simulations studying both the evolution of rising tubes within the convection zone and the process of flux emergence. From these simulations we know that the field of a flux bundle must be intertwined or twisted if it is to rise through the convection zone without suffering from significant distortion and shredding (Moreno-Insertis \& Emonet 1996; Dorch \& Nordlund 1998). We also know that when the field reaches the surface, there must be a negative gradient in its strength with increasing height if a magnetic buoyancy instability is to occur and emergence ensue.

The emergence process itself has been studied using both flux sheets and tubes, which are placed a short distance under the surface and rise either under buoyancy or via an imposed initial velocity. However, subsurface convective flows have been shown to distort flux sheets and twisted and untwisted magnetic flux tubes (Fan et al. 2003; Abbett et al. 2004). Hence, these emergence models have come under criticism for using an initial field structure which is too organised and, therefore, would not be expected to occur naturally in the Sun. We aim to create a more complex subsurface field through the interaction of two flux tubes within the solar interior. Dahlburg et al. (1997) observe a filamentary structure in the flux tubes of their simulation after they reconnect in a low- $\beta$ environment. The authors conclude that should reconnection occur between flux tubes in the convection zone, the emerging tubes are likely to have a fine scale internal structure too. We will study the subsequent emergence of our flux system and aim to provide an insight into how 
the structure of the field below the surface can manifest itself in atmospheric observations.

There have been multiple simulations that find reconnection occuring between two flux tubes. Fan et al. (1998) considers the rising motion of two parallel buoyant flux tubes in the convection zone, which are separated by both a horizontal and vertical distance. They find the lower tube can be drawn into the wake of the upper tube as they both rise and, eventually, the tubes will merge with dissipation of oppositely directed field components occurring at the interface between the tubes. The other simulations place more focus on studying the reconnection process between two interacting flux tubes but they have all been carried out in a low plasma- $\beta$ environment, with an aim to understanding whether the event provides the trigger for flares in the Sun's atmosphere. The most comprehensive study of reconnecting flux tubes is carried out by Linton et al. (2001), who classified the interactions of the tubes into four distinct classes dependent upon the orientation of the tubes to each other. Unlike Fan et al. (1998), the flux tubes are placed one above the other in these simulations and this is the model we choose to follow for our tubes in the convection zone.

There are significant differences between the simulations of our paper and the other "reconnecting tubes" studies (see Linton et al. 2001; Linton 2006, and references therein). Unlike the work of Linton, we will use the buoyancy of one of the tubes to initialise the interaction between the tubes rather than imposing a stagnation point flow to drive the two tubes together. The reconnection in our models will occur within the solar interior and will, therefore, be within a high plasma- $\beta$ environment. These differences will enable us to confirm the general nature of some of the results of previous colliding flux tube simulations. Finally, we will examine the effects of reconnection on the structure of emerging flux, an event that has not previously been studied.

Our paper is structured as follows: Sect. 2 will introduce the numerical code used for the simulations and explain the setup of our model with regard to the background stratification and the two tubes. Section 3 will present the results of the simulations looking at both the pre- and post-emergence evolution of the flux systems. In this section, we will also discuss our results in the context of existing literature. Section 4 will present our conclusions.

\section{Experiment setup}

\subsection{Equations}

The simulation results presented in this paper were obtained by solving the three-dimensional, time-dependent, resistive MHD equations numerically. These equations are:

$$
\begin{aligned}
& \frac{\partial \rho}{\partial t}=-\nabla \cdot(\rho \boldsymbol{v}), \\
& \frac{\partial(\rho \boldsymbol{v})}{\partial t}=-\nabla \cdot(\rho \boldsymbol{v} \boldsymbol{v}+\hat{\tau})-\nabla p+\rho \boldsymbol{g}+\boldsymbol{J} \times \boldsymbol{B}, \\
& \frac{\partial e}{\partial t}=-\nabla \cdot(e \boldsymbol{v})-p \nabla \cdot \boldsymbol{v}+Q_{\mathrm{Joule}}+Q_{\mathrm{visc}}, \\
& \frac{\partial \boldsymbol{B}}{\partial t}=-\nabla \times \boldsymbol{E}, \\
& \boldsymbol{E}=-\boldsymbol{v} \times \boldsymbol{B}+\frac{\boldsymbol{J}}{\sigma} \\
& \boldsymbol{J}=\nabla \times \frac{\boldsymbol{B}}{\mu}
\end{aligned}
$$

$p=\rho T \frac{\mathcal{R}}{\tilde{\mu}}$,

with density $\rho$, velocity $\boldsymbol{v}$, viscous stress tensor $\hat{\tau}$, gas pressure $p$, acceleration due to gravity $\boldsymbol{g}$, electric current density $\boldsymbol{J}$, magnetic field $\boldsymbol{B}$, thermal energy per unit volume $e=p /(\gamma-1)$, Joule dissipation $Q_{\text {Joule }}$, viscous dissipation $Q_{\text {visc }}$, electric field $\boldsymbol{E}$, electrical resistivity $\sigma$, magnetic permeability $\mu$, temperature $T$, gas constant $\mathcal{R}$, average atomic mass per particle $\tilde{\mu}$, respectively. $\gamma$ is the ratio of specific heats and is taken to be $5 / 3$.

\subsection{Numerical code}

Following the experiments of Archontis et al. (2004), we use a 3-dimensional code developed by Nordlund \& Galsgaard (1997). The MHD equations are solved on staggered grids using a sixth order accurate method to evaluate spatial derivatives and a fifth order interpolation method for shifting variables between the different grids. An explicit third order predictor-corrector method is used to advance the solution in time.

An integration box of $148 \times 160 \times 218$ points is used to model the dimensionless domain $(-60,60) \times(-70,70) \times$ $(-52,40)$ in the $(x, y, z)$ co-ordinate system. On the Sun this corresponds to a domain of $20.4 \mathrm{Mm} \times 23.8 \mathrm{Mm} \times 15.6 \mathrm{Mm}$. Uniform grid spacing is employed in the horizontal plane but the grid is stretched in the vertical direction. The numerical resolution is highest over the region $-33<z<-9$, which includes the initial locations of the tubes' axes and the region where we expect the reconnection to be initialised. At its smallest and largest, the grid spacing represents a physical distance of $4.77 \times 10^{4} \mathrm{~m}$ and $2.55 \times 10^{5} \mathrm{~m}$ respectively.

We operate periodic boundaries in the horizontal directions and closed boundaries at the top and bottom of the domain. Additionally, we include damping regions on the closed boundaries to limit the reflection of waves off them.

\subsection{Background stratification}

We begin by defining a background stratification comprising of four horizontal layers initially in hydrostatic equilibrium. In the base of the computational domain, $-52 \leq z<0$, is an adiabatic region with a linearly increasing temperature profile with depth and this represents the solar interior, extending to a depth of $-8.84 \mathrm{Mm}$ below the solar surface. Above the solar interior, there are two isothermal regions. The lower isothermal region resides within $0 \leq z \leq 10$ and corresponds to a photosphere with thickness $1.7 \mathrm{Mm}$. The upper isothermal region lies within $20 \leq z \leq 40$ and represents the first $3.4 \mathrm{Mm}$ of the corona.

The temperature of the corona, $T_{\mathrm{c}}$, is chosen to be 150 times greater than that of the photosphere, $T_{\mathrm{ph}}$. The region between the photosphere and the corona is termed the transition region and is characterised by a rapidly increasing temperature with height following the power law

$T(z)=T_{\mathrm{ph}}\left(\frac{T_{\mathrm{c}}}{T_{\mathrm{ph}}}\right)^{\left(z-z_{\mathrm{tr}}\right) /\left(z_{\mathrm{c}}-z_{\mathrm{tr}}\right)}$,

where $z_{\text {tr }}=10$ and $z_{\mathrm{c}}=20$.

At the photospheric base, we define the dimensionless pressure scale-height, temperature and density to be $H_{\mathrm{ph}}=1, T_{\mathrm{ph}}=1$ and $\rho_{\mathrm{ph}}=1$ respectively. The actual physical values of these parameters are found by multiplying the non-dimensional quantities by $H_{\mathrm{ph}}=1.7 \times 10^{5} \mathrm{~m}, T_{\mathrm{ph}}=5.6 \times 10^{3} \mathrm{~K}$ and $\rho_{\mathrm{ph}}=3 \times$ $10^{-4} \mathrm{~kg} \mathrm{~m}^{-3}$. Using these dimensional photospheric values we 
define a photospheric gas pressure unit $p_{\mathrm{ph}}=1.4 \times 10^{11} \mathrm{erg} \mathrm{m}^{-3}$, a velocity unit $V \equiv\left(p_{\mathrm{ph}} / \rho_{\mathrm{ph}}\right)^{1 / 2}=6.8 \times 10^{3} \mathrm{~m} \mathrm{~s}^{-1}$, a time unit $t_{\mathrm{ph}}=25 \mathrm{~s}$ and a magnetic field unit $B_{\mathrm{ph}}=1.3 \times 10^{3}$ Gauss. Surface gravity is $2.7 \times 10^{2} \mathrm{~m} \mathrm{~s}^{-2}$ and $\tilde{\mu}=1$.

\subsection{Magnetic flux tubes}

We define two horizontal flux tubes in the solar interior. In cylindrical coordinates $\left(r, \theta, y^{\prime}\right)$, the magnetic field of each tube is given by

$$
\begin{aligned}
& B_{r}=0 \\
& B_{\theta}=\alpha r B_{y^{\prime}} \\
& B_{y^{\prime}}=B_{0} \exp \left(-r^{2} / R^{2}\right) .
\end{aligned}
$$

The equations ensure the magnitude of the field falls with distance from the axis following a Gaussian profile. $B_{0}$ is the magnitude of the field at the axis and $R$ specifies the radius at which the axial field measures $37 \%$ of $B_{0}$. This magnetic field profile results in the field strength being exactly zero at $r=\infty$ but the strength actually becomes computationally negligible at some finite radius. We call this finite radius the outer radius of the tube. The radian angle through which the fieldlines rotate over a distance of one unit in the axial direction is more commonly refered to as the twist of the fieldlines about the axis. It is defined as $\Phi=B_{\theta} / r B_{y^{\prime}}$ and, fro (10) and (11), we have $\Phi=\alpha$.

The axis of the upper tube is situated at a depth of $z=-10$ and the axial direction, $y^{\prime}$, is set to be the Cartesian coordinate $y$. The azimuthal and radial coordinates of the tube are, therefore, comprised of the Cartesian $x$ and $z$ directions, with $x=r \cos \theta$ and $z=-10+r \sin \theta$. We set $B_{0}=3$ and choose the tube to be in mechanical equilibrium with its environment, thus it is slightly cooler than the surroundings. The axis of the lower tube is situated at $z=-30$, with $B_{0}=15$. This tube is chosen to be in thermal equilibrium with the environment so that it will be buoyant and, therefore, rise and interact with the upper tube. Following the experiments of Fan (2001) and Archontis et al. (2004), we wish to encourage the formation of an $\Omega$-shaped loop, thus we decrease the temperature away from the central portion of the lower tube, following a Gaussian profile.

We choose $\alpha=-0.4$ and $R=2.5$ for both of the tubes. The negative value for $\alpha$ results in both of the tubes having left hand twist about the axis. We leave the study of differently twisted tubes for a later date. The larger value of $B_{0}$ for the lower tube gives the axial field a strength of 5.52 at a radius $R$, in comparison with 1.1 at the same radius for the upper tube. This means that the radius at which the field becomes negligible for the lower tube is greater than that of the upper tube. Hence, the pitch of the fieldlines at this larger outer radius of the lower tube is also greater. The plasma- $\beta$ at the axes of the upper and lower tubes measures 11.13 and 4.86, respectively, at $t=0$.

One aspect of our experiments is to demonstrate that, when the initially separate flux domains of the two tubes come into contact with each other, reconnection will occur if the fields of the tubes are suitably aligned. Therefore, the orientation of the lower tube, in reference to the axial direction of the upper tube, will be varied to identify how the changing orientation of the flux systems alters any reconnection and resulting complexity of the magnetic field.

As discussed above, we fix the axial direction of the upper tube to be in the $y$ direction. In model 1 we choose the axis of the lower tube to be parallel to the axis of the upper tube and, thus,
Table 1. The relationship between axial and transverse components and the Cartesian coordinate system for tubes $\mathrm{ft} 1$ and $\mathrm{ft} 2$ in the three models. The transverse component is proceeded by \pm since the transverse

\begin{tabular}{|c|c|c|c|c|}
\hline & \multicolumn{2}{|r|}{$\overline{\mathrm{fft} 1}$} & \multicolumn{2}{|r|}{$\mathrm{ft} 2$} \\
\hline & axial & transverse & axial & transverse \\
\hline Model 0 & - & - & $y$ & $\pm x$ \\
\hline Model 1 & $y$ & $\pm x$ & $y$ & $\pm x$ \\
\hline Model 2 & $y$ & $\pm x$ & $-x$ & $\pm y$ \\
\hline
\end{tabular}
directions of the field at the top and bottom of each tube will be exactly opposite to each other.

the azimuthal and radial coordinates are the same as for the upper tube. In model 2 we choose the axes of the two tubes to be orthogonal, with the axis of the lower tube $\left(y^{\prime}\right)$ in the $-x$ Cartesian direction and, thus, its azimuthal and radial components will be prescribed by $y=r \cos \theta$ and $z=-30+r \sin \theta$ components of the Cartesian geometry.

In addition to these two cases, we perform a reference experiment to check the evolution and emergence pattern of the lower tube in the absence of the upper tube and this will be referred to as model 0 . The positioning of this single flux tube is the same as that of the lower tube in model 1.

For clarity and brevity we will refer to the upper flux tube as $\mathrm{ft} 1$ and the lower flux tube as $\mathrm{ft} 2$ in the remainder of the paper. When describing the components of the field we will use the terms axial and transverse to allow for comparison between the models since the axes of the tubes and the horizontal component of the azimuthal field actually have different Cartesian directions. Table 1 summaries the Cartesian components prescribing the axial and transverse field components for $\mathrm{ft} 1$ and $\mathrm{ft} 2$ in all three models.

\section{Results}

We begin by giving an overview of the results from model 0 , which includes only $\mathrm{ft} 2$. Independent of the orientation of the lower tube, all of the models would show this evolution in the absence of $\mathrm{ft} 1$.

Under buoyancy, the central portion of the tube begins to rise and compressional effects at the front of the tube cause the orientation of the fieldlines to turn more towards the transverse direction. Due to continual small-scale horizontal expansion, the tube experiences a decrease in both the strength of its axial field component and its internal density. The tube eventually becomes overdense whilst still in the solar interior but keeps rising due to the marginally stable nature of the surrounding environment to convective motions.

Once the tube front reaches the photosphere it experiences a rapid deceleration due to the strongly subadiabatic nature of the photosphere. However, through the nonlinear development of a magnetic buoyancy instability, the tube advances into the atmosphere and undergoes both horizontal and vertical expansion. The axis of the tube remains confined to the solar interior during the limited time of the experiment. (Arber, private communication, has shown that the axis will eventually emerge given time.)

This general evolution of a rising flux tube and its emergence into both magnetised and unmagnetised atmospheres is well known (see Murray et al. 2006, and references therein for a more complete explanation). The specific measurements obtained from model 0 will be used to understand how the introduction of $\mathrm{ft} 1$ modifies the evolution of $\mathrm{ft} 2$ in the solar interior and the atmosphere. 


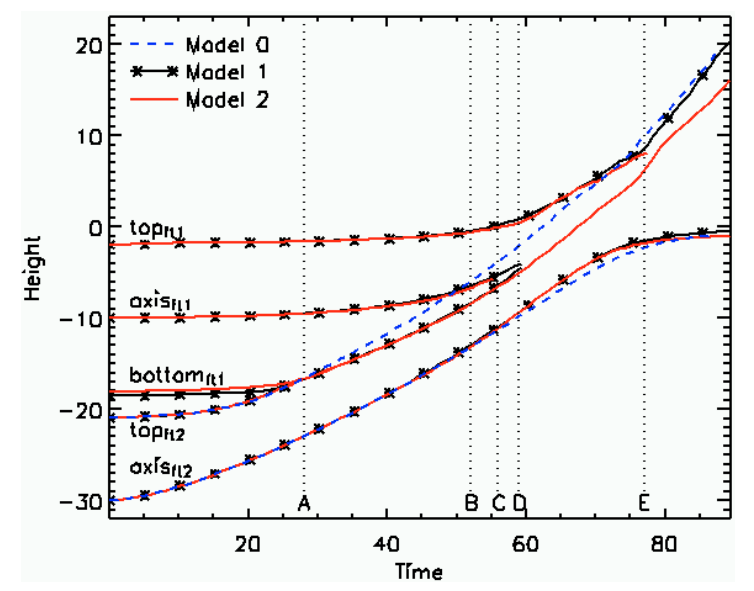

Fig. 1. Height of various points of the flux tubes over time for model 0 (blue), model 1 (stars) and model 2 (red). The top, axis and (unreconnected) bottom of $\mathrm{ft} 1$ are tracked and the top and axis of $\mathrm{ft} 2$ are tracked. The vertical lines and labels describe the following: A $(t=28)-$ first contact of the two tubes; $\mathrm{B}(t=52)-$ axis of $\mathrm{ft} 2$ begins to rise faster in models 1 and 2 than model 0 ; $\mathrm{C}(t=56)$ - axis of $\mathrm{ft} 1 \mathrm{re}-$ connects in model 2; $\mathrm{D}(t=59)$ - axis of $\mathrm{ft} 1$ reconnects in model 1 ; $\mathrm{E}(t=76)$ - magnetic field of $\mathrm{ft} 2$ becomes uppermost flux in model 2 .

\subsection{Rise of the tubes prior to contact}

In all three models, the only variation in $\mathrm{ft} 2$ is the orientation of the axial (and therefore transverse) component of the tube's field with respect to the Cartesian coordinate system. Hence, one would expect the initial evolution of $\mathrm{ft} 2$ to be similar in all models, with only significant changes occurring once $\mathrm{ft} 2$ is in close proximity to ft1. In Fig. 1, we track the evolution of the top, axis and bottom of $\mathrm{ft} 1$ and the top and axis of $\mathrm{ft} 2$ along the central vertical line of the domain at $(x=0, y=0)$. Thus, we see that $\mathrm{ft} 2$ does indeed undergo the same evolution in all three models until $t=28$. As can be seen from label A in Fig. 1, at this time the top of $\mathrm{ft} 2$ comes into contact with the bottom of $\mathrm{ft} 1$ in models 1 and 2.

For all three models we find the same behaviour for many of the physical quantities over the first 28 time units. Thus, the qualitative description of $\mathrm{ft} 2$ given at the beginning of Sect. 3 is quantitatively the same in all three models. However, we do find variations in the changing orientation of the fieldlines of $\mathrm{ft} 2$ in models 1 and 2 compared to model 0 . The field at the front of ft 2 increases away from the axial field direction by $\sim 10$ degrees during this early rise phase in models 1 and 2, such that it is practically in the transverse direction. In model 0 , we find only a $\sim 5$ degree increase during this time period. Hence, we conclude that the compression of the field at the front of $\mathrm{ft} 2$ is caused not only by the enhanced buoyancy in the centre of the tube but also by the presence of $\mathrm{ft} 1$. The mass and rigid magnetic structure of $\mathrm{ft} 1$ act to slow the advance of the front of $\mathrm{ft} 2$ while the axis of $\mathrm{ft} 2$ continues to evolve as though $\mathrm{ft} 1$ were not present, thus resulting in the additional compression.

In models 1 and 2, ft1 first starts to move upwards at $t=3$. The initial movement is caused by the launch of an upgoing compression wave at the beginning of the simulation due to the sudden motion of $\mathrm{ft} 2$ and the reaction of the surrounding plasma. This wave has previously been discussed by Archontis et al. (2004), who found that whilst the wave has small amplitude in the solar interior it steepens significantly in the atmosphere to become a shock wave. We find that this wave has no effect on $\mathrm{ft} 1$ other than to provide it with a small amount of positive vertical momentum. The further acceleration of $\mathrm{ft} 1$ occurs when the plasma above $\mathrm{ft} 2$ is displaced upwards by the buoyancy of this lower tube.

When the two tubes come into magnetic contact with each other at $t=28$, the location of the bottom of $\mathrm{ft} 1$ and the top of $\mathrm{ft} 2$ becomes identical. The two touching surfaces act as one and, due to the differing field orientations of the two flux systems across the interface, a current sheet forms here. Reconnection between the two tubes ensues and the exact properties of this process are discussed in Sects. 3.2 and 3.3.

\subsection{Current sheets and reconnection}

Ft 2 was chosen to be most buoyant in its central portion $(x=$ $0, y=0)$ and, therefore, it is in this region that the flux systems of $\mathrm{ft} 1$ and $\mathrm{ft} 2$ first come into contact with each other at $t=28$. The differences in the initial setup between models 1 and 2 now begin to play a role.

For model 1, the axes of the two tubes are in the same direction but, due to the tubes having the same sense of twist, the magnetic fields at the bottom of $\mathrm{ft} 1$ and top of $\mathrm{ft} 2$ have oppositely directly transverse field components. For model 2, on the other hand, both the axial and transverse magnetic field components of the two tubes are differently directed at the contact interface. This results in the field at the bottom of ft1 being virtually anitparallel to the field at the top of $\mathrm{ft} 2$ in model 2.

The nonparallel magnetic fields at the bottom of $\mathrm{ft} 1$ and the top of $\mathrm{ft} 2$, for both models 1 and 2 , cause a current sheet to build up between the two tubes. The current sheet is centred about the point of first contact $(x=0, y=0)$. The simulations of Galsgaard et al. (2005) found that, when two differently directed flux systems come into contact with each other, the current sheet that forms between them is orientated at an angle between the differently directed field components, with the exact angle being determined by the magnitude of the non-reconnecting field component on either side of the sheet. The current sheets we see agree with this finding. When the tubes are parallel, as in model 1 , the current sheet takes the form of a thin ribbon that is parallel to the axial direction of the flux tubes, Fig. 2(left). When the tubes lie orthogonal to each other, as in model 2, the current sheet is much shorter and lies orthogonal to the practically antiparallel fields of the two touching systems, Fig. 2 (right).

Reconnection between the magnetic fields of $\mathrm{ft} 1$ and $\mathrm{ft} 2$ begins at $t=30$ for model 1 and $t=35$ for model 2 , following the same general pattern in both models. The field in the current sheet, at the interface of the two tubes, reconnects such that the fieldlines of $\mathrm{ft} 1$ become wrapped around $\mathrm{ft} 2$ and vice versa. Reconnected fieldlines are ejected from the current sheet by flows, thus ensuring the reconnection region does not become clogged and reconnection can continue. If this reconnection was occurring in a low plasma- $\beta$ environment, we would expect to find jets with velocities below the order of the local Alfvén speed directed along fieldlines (Archontis et al. 2005). However, the jets are obscured by both the high plasma- $\beta$ surroundings, where the Alfvén speed is small compared to the sound speed, and the general motion of the tubes and surrounding plasma. The continued rise of ft 2 under its own buoyancy ensures that the current sheet does not diffuse away between the two systems but is pushed upwards over time. As time progresses, the height of the strongest point in the current sheet matches the heights of the top of $\mathrm{ft} 2$ and bottom of $\mathrm{ft} 1$, shown in Fig. 1.

The reconnection of fieldlines proceeds from the outside of each tube moving towards their axes. The rate at which the reconnection advances towards the axis of an individual tube is dependent upon the amount of flux lying between the outer edge 

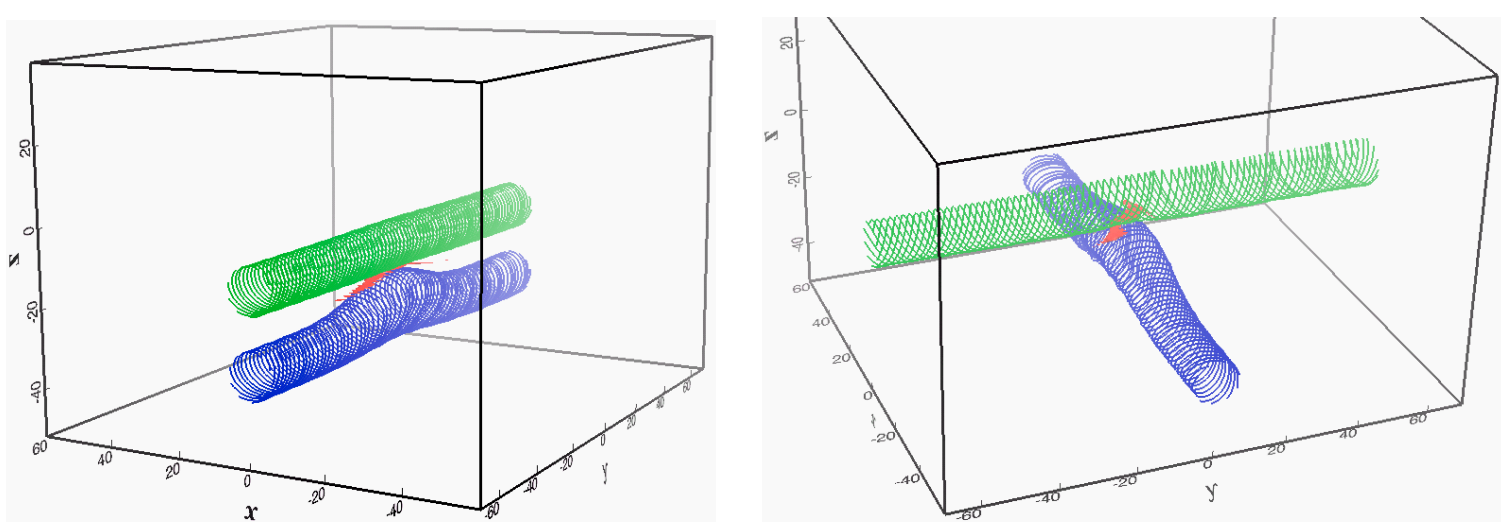

Fig. 2. Outer fieldlines of $\mathrm{ft} 1$ (green) and $\mathrm{ft} 2$ (blue) for model 1 at $t=30$ (left) and model 2 at $t=34$ (right). The red isosurfaces show the current sheet, calculated as $|J| /|B|$, between the two flux systems at a level of 5.6 and 4.9 for models 1 and 2 , respectively.
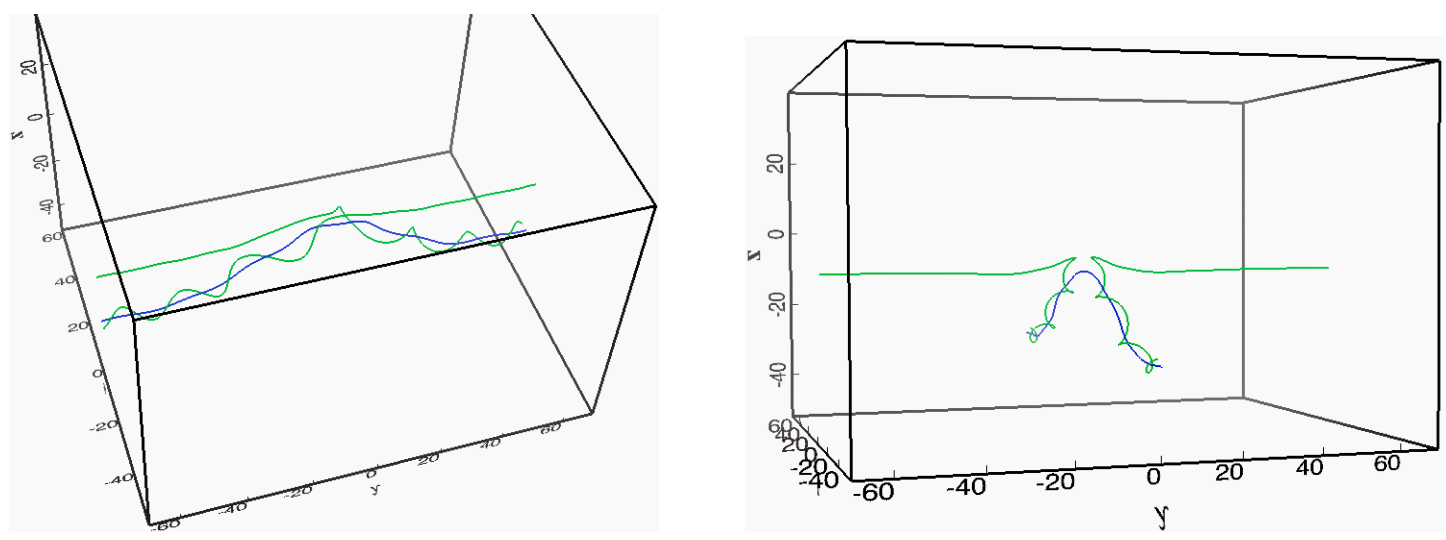

Fig. 3. The axes of both $\mathrm{ft} 1$ (green) and $\mathrm{ft} 2$ (blue) for model 1 at $t=59$ (left) and model 2 at $t=56$ (right). In both models the axis of ft 1 has reconnected with twisted fieldlines lying above the axis of $\mathrm{ft} 2$ and, thus, the axis of $\mathrm{ft} 1$ has become wrapped around the axis of $\mathrm{ft} 2$.

of the tube and its centre, measured along a radial line. Although the strength of $\mathrm{ft} 2$ 's magnetic field decreases overall as the tube rises, when the tubes make contact the field strength on the axis of $\mathrm{ft} 2$ is still 3.3 times larger than that of $\mathrm{ft} 1$ in both models 1 and 2 . Hence, the amount of flux in the vertical column lying between the axis of $\mathrm{ft} 2$ and its approximate front edge is much greater than that lying between the bottom edge of $\mathrm{ft} 1$ and its axis. Thus, the axis of $\mathrm{ft} 1$ is drawn into the reconnection region when the axis of $\mathrm{ft} 2$ is still surrounded by some of its original fieldlines. Therefore, the axis of $\mathrm{ft} 1$ reconnects with twisted fieldlines of $\mathrm{ft} 2$ and becomes wrapped around the axis of $\mathrm{ft} 2$, as shown in Fig. 3. Taking measurements along the central vertical line of the domain $(x=0, y=0)$, it is no longer possible to detect the axis of $\mathrm{ft} 1$ once its reconnection occurs at $t=59$ and $t=56$ for models 1 and 2 respectively. This is shown by the disappearance of the lines representing $\mathrm{ft} 1$ 's axis for both models 1 and 2, labelled D and C in Fig. 1. The greater flux of $\mathrm{ft} 2$ results in the reconnection region never reaching its axis and thus the axis persists for the length of the simulation.

The rise of a flux tube in a domain with an overlying flux of significant strength has been considered by Dorch (2006) and Galsgaard et al. (2005) for the convection zone and corona respectively. They found that the inclusion of the flux sheet slows the rise of the tube, regardless of whether reconnection occurs between the two magnetic systems. This slow down is due to the presence of an increased downward acting tension force associated with the "dented" flux sheet. There is a similar increase in the tension force when the horizontal sheet is replaced by a flux tube and we find a corresponding slower rise of the top of $\mathrm{ft} 2$ when $\mathrm{ft} 1$ is included in models 1 and 2.

The deceleration of the top of $\mathrm{ft} 2$ causes the field between the front and axis of $\mathrm{ft} 2$ to become compressed. There is a consequential increase in the upward directed magnetic pressure force above the axis. Thus, the axis experiences a slight acceleration in models 1 and 2 at $~ 52$ time units, labelled B in Fig. 1. However, in model 0 there is less compression of the field situated above the axis and, therefore, the axis rises more slowly. Regardless of this acceleration, we find that in all of the models the axis of $\mathrm{ft} 2$ remains within the solar interior within the time of the experiments. Measurements of field strength, density and gas pressure identify that the presence of $\mathrm{ft} 1$ has little effect on the evolution of the axis of $\mathrm{ft} 2$.

During the reconnection process, $\mathrm{ft} 2$ is still rising through the solar interior. For the field of $\mathrm{ft} 2$ that does not undergo reconnection, the magnetic field strength, density and gas pressure have the same evolution with respect to time as they would if $\mathrm{ft} 1$ were not there, as shown by the evolution of the axis of $\mathrm{ft} 2$. When the first magnetic flux appears at the photosphere, there is still reconnection occurring in models 1 and 2 . The effects of this and the emergence process of all three models will be discussed in Sect. 3.4.

\subsection{Connectivity and reconnected flux}

To determine how the reconnection proceeds and differs in each of the models, we consider how the connectivity of fieldlines 

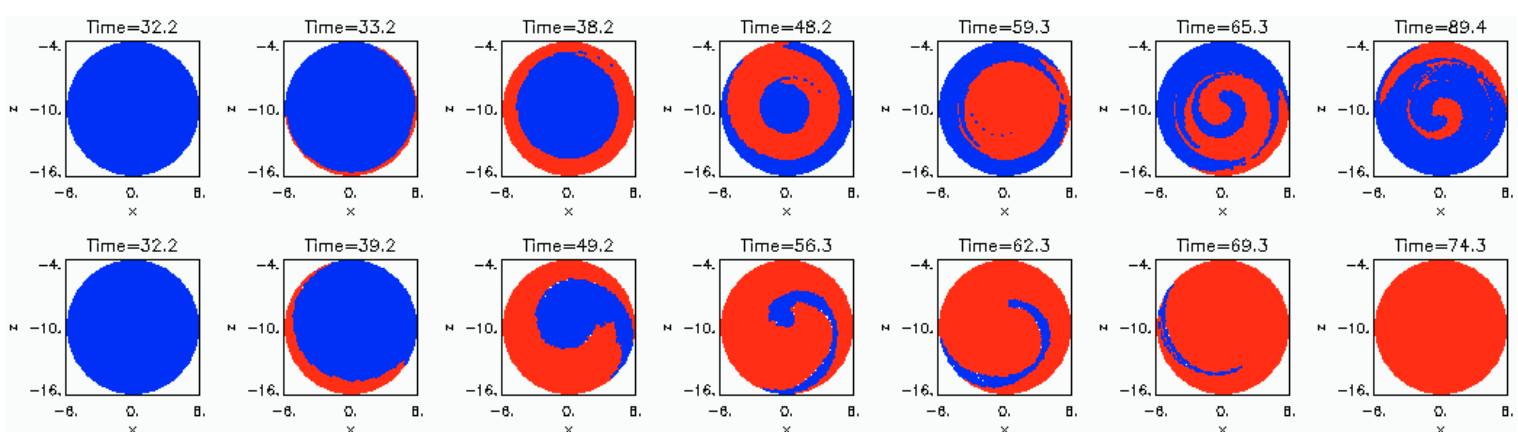

Fig. 4. Fieldlines traced from one end of $\mathrm{ft} 1$ and coloured according to where each fieldline ends. Blue fieldlines start at one end of ft 1 and end at the other end of $\mathrm{ft} 1$ and red fieldlines start at one end of $\mathrm{ft} 1$ and end in either end of $\mathrm{ft} 2$. The top and bottom rows of images are for models 1 and 2 respectively.

changes over time. We trace a large number of fieldlines (4881) from starting points that reside in one end of $\mathrm{ft} 1$ and calculate whether the endpoints lie in $\mathrm{ft} 1$ or $\mathrm{ft} 2$, a technique previously used by Parnell \& Galsgaard (2004). The start points are divided between 39 concentric circles whose locus is $(-10,0)$ in the $(z, x)$ plane at $y=-70$, where the $39^{\text {th }}$ circle corresponds to a radius of $r_{1}=5.85$ units.

Before using this technique, we have analysed the velocities in the footpoints of $\mathrm{ft} 1$. For both models, we find no large scale movement or signs of rotation in the start or end points of the fieldlines of $\mathrm{ft} 1$. We cannot state emphatically that we trace the same fieldline over time when using the same starting point but, as we will see below, the general evolution of the footpoints gives a definite guide for where reconnection occurs and how the field connectivity changes.

The results of the connectivity technique are shown in Fig. 4 for model 1 (top row) and model 2 (bottom row). Fieldlines whose end points lie within a radius $1.5 r_{1}$ of the initial locus on the $y=70$ plane are deemed to be fieldlines of $\mathrm{ft} 1$ and their start points are coloured blue. If a fieldline's end point lies within a suitable radius of the axis of $\mathrm{ft} 2$ on either the $\pm y$ planes for model 1 or the $\pm x$ planes for model 2 , then the fieldline is considered to have reconnected and its start point is coloured red. Fieldlines whose end points meet none of these criteria are indicated by white start points but, as illustrated by Fig. 4, these occurrences are rare.

Figure 4 shows very different connectivity patterns for models 1 and 2, indicating that the reconnection proceeds in a different manner in each model. Additionally, Fig. 5 demonstrates that whilst the percentage of reconnected ft 1 flux increases continually in model 2 , there is an oscillating variation of reconnected flux with time for model 1.

\subsubsection{Model 1 - parallel tubes}

As previously stated, the current sheet formed between the two flux tubes in model 1 is long and thin, lying in the same direction as the axes of the two tubes. It is the outermost fieldlines of $\mathrm{ft} 1$ which will experience reconnection first as these are the first ones to come into contact with the field of $\mathrm{ft} 2$. For the fieldlines on this outer radius of $\mathrm{ft} 1$, reconnection occurs first for the fieldlines passing through the central point of the current sheet $(x=0, y=0)$ above the most buoyant portion of $\mathrm{ft} 2$, as shown by Fig. 6. Over time, the location of the reconnection for the outer set of fieldlines moves away from the central point of the current sheet and migrates towards its flanks. Once the reconnection of fieldlines on a particular radius moves towards the flanks of the current sheet, reconnection will begin for the next

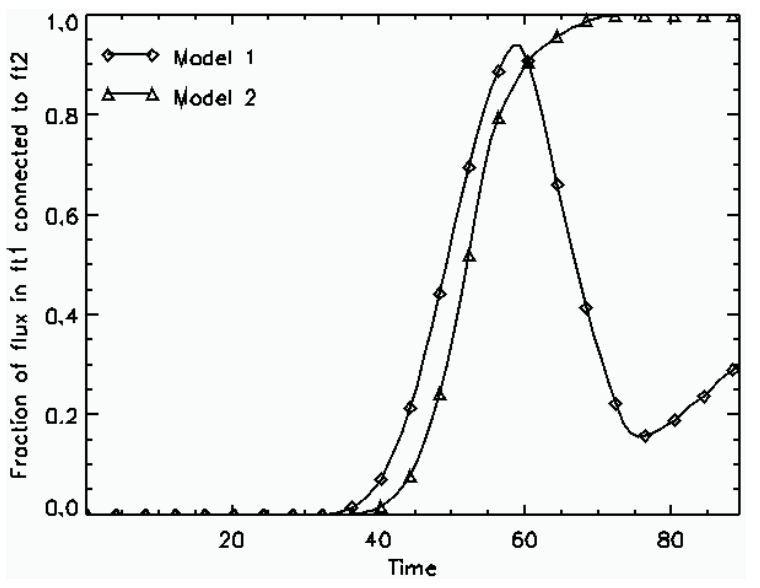

Fig. 5. Fraction of the magnetic flux associated with the start points of $\mathrm{ft} 1$ that have endpoints in $\mathrm{ft} 2$ as a function of time for models 1 (diamonds) and 2 (triangles).

inner radius of fieldlines, again starting in the centre of the current sheet, Fig. 7. And so the reconnection continues, for each radius starting in the middle of the current sheet and migrating to the flanks.

The fieldlines are highly twisted on the edge of $\mathrm{ft} 1$, thus all fieldlines on a certain radius will reconnect in the current sheet over a short period of time and a short length of the current sheet. At $t=38.2$, Fig. 4 shows all of the fieldlines on the outer radii have reconnected with fieldlines from $\mathrm{ft} 2$ and thus their endpoints are now in $\mathrm{ft} 2$. However, $\mathrm{ft} 2$ is buoyant along a large portion of its length, with lessening degree away from $(x=0, y=0)$. Thus, the current sheet continues to extend in the axial direction as more of the outer fieldlines of each tube are brought into contact with each other. Fieldlines that have already experienced reconnection find themselves passing through the more recently created flanks of the current sheet. Hence, they undergo multiple and continual changes of connectivity during our experiment, Fig. 6. This is visible in Fig. 4 from the changing spiraling patterns of colour at $t=65.3$ and $t=89.4$.

As the reconnection proceeds, the radius of the fieldlines, which are experiencing their first reconnection, migrates in towards the axis of $\mathrm{ft} 1$. The number of rotations of the fieldlines about the axis reduces as their distance from the axis reduces, according the constant twist definition. Thus, the fieldlines of inner radii will pass through the current sheet less times, with the inner most passing through only once. Figure 4 shows that once the central fieldlines have reconnected once they do not change their connectivity again within the time of the experiment. 

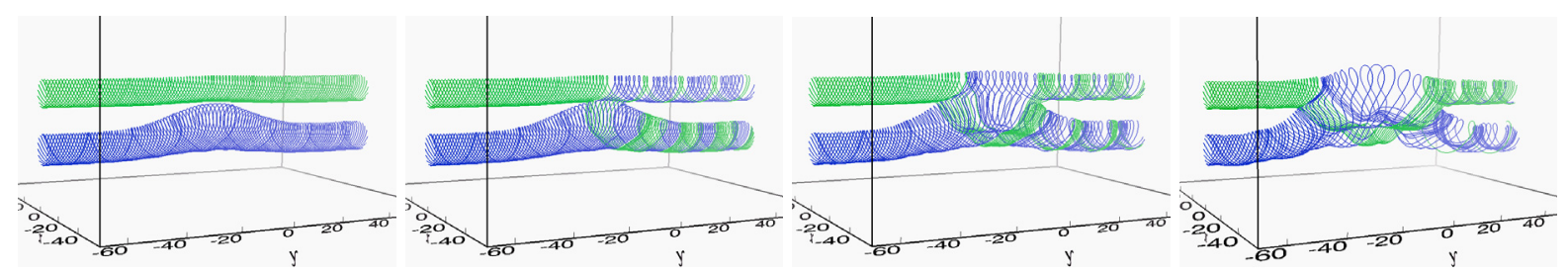

Fig. 6. For model 1, twisted outer fieldlines of $\mathrm{ft} 1$ (green) and $\mathrm{ft} 2$ (blue) at $t=35,37,48$ and 65 (left to right). Reconnection for these radii begins in the centre of the current sheet and over time moves out towards the flanks of the sheet.

At $t=59$, the time at which the axis of $\mathrm{ft} 1$ undergoes reconnection, we find that $94 \%$ of the flux belonging to $\mathrm{ft} 1$ has reconnected into $\mathrm{ft} 2$. Once the axis of $\mathrm{ft} 1$ has been reconnected, the central part of the current sheet diffuses away since the field above the axis of $\mathrm{ft} 1$ is orientated in the same direction as the field of $\mathrm{ft} 2$ it is coming into contact with.

The continuous reconnection of fieldlines gives rise to the oscillatory changes in the percentage of reconnected flux in Fig. 5. The fieldlines with start points nearer the locus of the circle have a greater amount of flux associated with them since the field strength increases with decreasing distance to the axis and, therefore, they influence the percentage of reconnected flux the most. These inner fieldlines are also the least twisted and have the greatest period of time between changes of connectivity. The initial period of increasing reconnected flux corresponds to reconnection of the inner circles for the first time, with the peak in the reconnecting flux occurring when the axis of $\mathrm{ft} 1$ changes connectivity. The decrease and subsequent increase takes place as these inner fieldlines reconnect for a second and third time respectively. We believe the oscillatory pattern of Fig. 5 would continue if the experiment ran for longer, with the oscillation period increasing due to the decreasing buoyancy of $\mathrm{ft} 2$ 's legs with distance from the initial, central reconnection location.

By the end of the simulation, $t=89$, the flux system in the centre of the domain is tube-like with fieldlines winding around the non-reconnecting core of $\mathrm{ft} 2$. The windings per unit length of the tube have reduced by a factor of 2 . These findings are inline with those of Linton et al. (2001) and Linton (2006) and indicate a merging action in progress. If the experiment was run for longer, we believe the central single flux tube region would be extended as reconnection continues, although it is unclear whether a complete merger along the entire lengths of the tubes would occur.

\subsubsection{Model 2 - orthogonal tubes}

For model 2, the current sheet is much shorter since the orientation of the tubes brings a smaller area of them into contact with each other. At the start of the reconnection period, the current sheet is almost entirely horizontal and the particular twist constant chosen for the tubes results in even the greatly twisted outer fieldlines only passing through the current sheet once and, therefore, experiencing only one potential reconnection site.

For each circle of start points, reconnection begins with the fieldlines in the central location of the current sheet and then moves to the fieldlines in contact with the flanks of the current sheet. Hence, reconnection spreads both clockwise and anticlockwise on each circle between $t=39.2$ and $t=49.2$ in Fig. 4. The reconnected fieldlines are moved out of the reconnection region in a direction parallel to the reconnecting components. However, the twisted nature of the field prevents the fieldlines from escaping far from the interaction site of the two tubes.

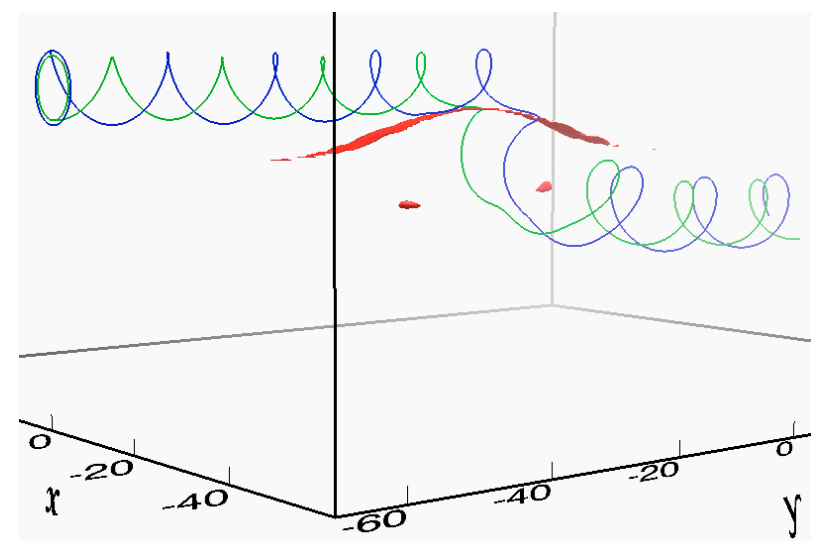

Fig. 7. For model 1 at $t=40$, the red isosurface indicates the current sheet at $|J| /|B|=5.0$. The outer blue fieldline, traced from the blue circle with a radius of 4.5 , is reconnecting in the flank of the current sheet while the inner green fieldline, traced from the green circle with a radius of 3.9, is reconnecting at the centre of the current sheet.

At the time when the axis of $\mathrm{ft} 1$ reconnects, $t=56,75 \%$ of the flux of $\mathrm{ft} 1$ has reconnected into $\mathrm{ft} 2$, Fig. 5. This is smaller than the level of reconnected flux experienced in model 1 by the time of its axis reconnection because the region of contact in model 2, and thus the current sheet, is much smaller. This allows fewer fieldlines to reconnect prior to the axis being reached. However, once the axis of $\mathrm{ft} 1$ has reconnected, the field above $\mathrm{ft} 1$ 's axis still has a differing orientation to the field of $\mathrm{ft} 2$ it is in contact with. Hence, the reconnection continues and the percentage of reconnected flux increases further. As can be seen from the isosurfaces of field magnitude in Fig. 8, the rise of $\mathrm{ft} 2$ causes $\mathrm{ft} 1$ to take on an $\Omega$ shape along its length and, thus, the current sheet between the two systems becomes more curved over time, though still ribbon like and with the same orientation.

The fieldlines of $\mathrm{ft} 1$ that reconnect in the centre of the current sheet belong to a special group of fieldlines. During their first reconnection, the majority of the axial and transverse components of these ft1 fieldlines were annihilated with the corresponding components of the $\mathrm{ft} 2$ fieldlines, leaving the vertical component as the most significant in size. The reconnection effectively "cut" these $\mathrm{ft} 1$ fieldlines and they lie on either side of $\mathrm{ft} 1$. Figure 8 (left) illustrates the post-reconnection fieldlines on one side of $\mathrm{ft} 1$, which are traced from the footpoint of $\mathrm{ft} 1$ and are coloured by their $B_{z}$ component. As the mass of $\mathrm{ft} 2$ continues to rise, these fieldlines become less curved with time and their $B_{z}$ component diminishes. However, the reduction in $B_{z}$ increases the significance of the small axial component, $B_{y}$, of the fieldlines and their orientation moves towards the axial direction as shown in Fig. 8 (right). The fieldlines become increasingly curved over the shoulder of ft 2 and the return of a field component oppositely 


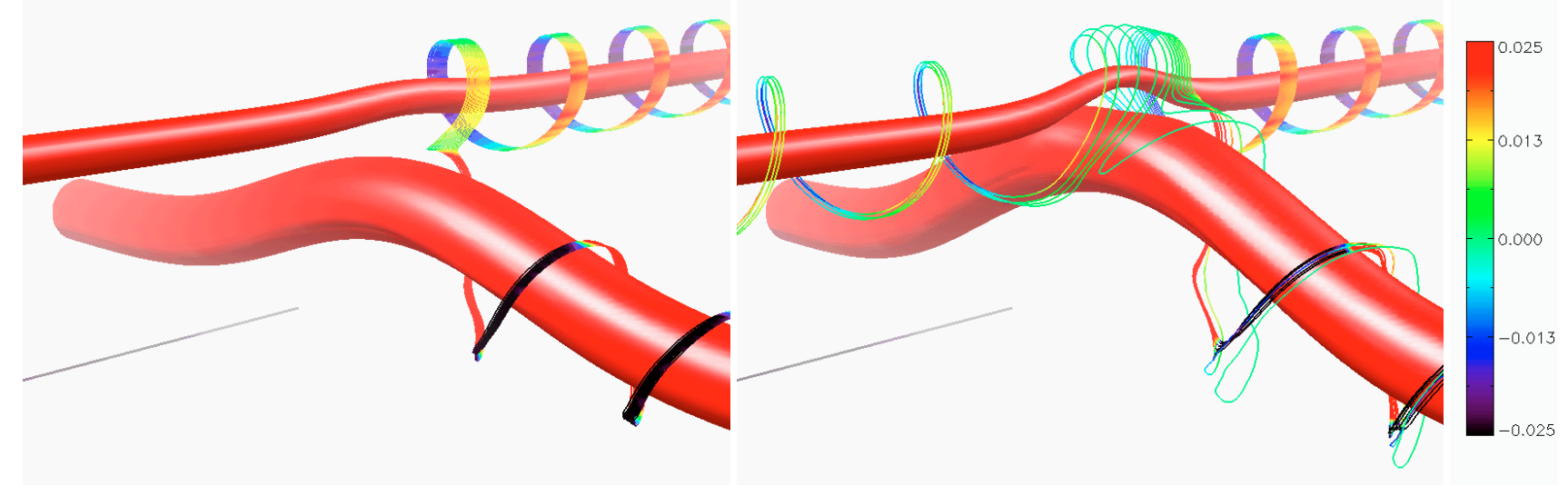

Fig. 8. For model 2, a selection of outer fieldlines traced from one end of $\mathrm{ft} 1$ at $t=37$ (left) and $t=50$ (right). The fieldlines are coloured along their length according to $B_{z}$ and the righthand colour bar indicates the scaling in use. The red isosurfaces give $|B|=2.0$.

directed to the surrounding field results in a second reconnection located in one flank of the current sheet. This returns the end points of the set of fieldlines with start points in $\mathrm{ft} 1$ to $\mathrm{ft} 1$.

This process of changing orientation and reconnection is replicated for the fieldlines on the other side of $\mathrm{ft} 1$. However, rather than the footpoints of the special group of fieldlines returning to $\mathrm{ft} 1$ through this secondary reconnection we find that the fieldlines that reconnect on one side of $\mathrm{ft} 1$ are the same fieldlines that will reconnect on the other side of $\mathrm{ft} 1$ and, thus they experience a total of three reconnections with their footpoints ultimately terminating in $\mathrm{ft} 2$. This is due to the specific twist of $\mathrm{ft} 1$ and radius of $\mathrm{ft} 2$. If the twist of $\mathrm{ft} 1 \mathrm{had}$ been less or the radius of $\mathrm{ft} 2$ has been greater we believe that, once the reconnection process finished, some fieldlines of $\mathrm{ft} 1$ would have had footpoints in $\mathrm{ft} 2$ whilst others would have had their footpoints returned to $\mathrm{ft} 1$.

If all of the fieldlines of $\mathrm{ft} 1$ reconnected only once we could expect to see symmetrical growth and slowdown in the rate of change of reconnected flux at the start and end of the reconnection phase respectively displayed in Fig. 5. This is because $\mathrm{ft} 2$ reconnects first with the weaker outer, then stronger inner and finally weaker outer field of $\mathrm{ft} 1$ as it "carves" its way to the surface. However, the reconnection slowdown occurs over a much longer period of time than the growth phase and this is as a result of the multiple reconnections of the select group of fieldlines.

All of the fieldlines associated with the 4881 chosen start points belong to one of two categories, either reconnecting once or three times and, therefore, when reconnection ends at $t=74.3$ all of the fieldlines have their end points in ft2, Fig. 4.

From the results of Linton (2006), we would expect this orientation of equal strength tubes to result in them performing a slingshot action due to their low level of twist. We certainly see the start of this interaction, with the fieldlines of ft 1 wrapping around the two legs of $\mathrm{ft} 2$ after the reconnection. However, once the reconnection ends we do not see a complete slingshot, which would have resulted in two differently connected and distinct tubes. This is because the tubes differ in strength by a factor of $\sim 3.3$ when they first come into contact with each other and the reconnection starts. For a complete slingshot it is necessary for the axis of $\mathrm{ft} 1$ to reconnect with the axis of $\mathrm{ft} 2$ but in our simulation this does not happen. Thus, we end up with the weaker tube hanging off the core of the stronger tube. This end state is also found by Linton (2006), whose tubes differed in strength by a factor of two.

\subsection{Emergence}

We must first establish the topology of the uppermost magnetic field prior to its arrival at the photosphere in models 1 and 2, before understanding how the emergence occurs. As discussed in Sect. 3.3, reconnection changes the footpoints of the fieldlines originally belonging to both $\mathrm{ft} 1$ and $\mathrm{ft} 2$. Prior to emergence this process occurs at the interface between $\mathrm{ft} 1$ and $\mathrm{ft} 2$, which is situated away from the top edge of $\mathrm{ft} 1$. When considering the $3 \mathrm{D}$ structure of the flux, it becomes increasingly difficult to distinguish between the two tubes in the centre of the domain as the reconnection advances. Although the field initially closest to the photosphere was that of $\mathrm{ft} 1$, reconnection means that these fieldlines could now belong to $\mathrm{ft} 1, \mathrm{ft} 2$, or have an endpoint situated in each. However, since reconnection occurs at the interface of $\mathrm{ft} 1$ and $\mathrm{ft} 2$ we know that the field segments at the top of the magnetic flux system, which originally belonged to $\mathrm{ft} 1$, remain unchanged and have approximately their original ft1 field strength. These segments are directly above the fastest rising portion of $\mathrm{ft} 2$, and, thus, it is this flux that will enter the photosphere first for models 1 and 2.

The flux at the photospheric plane $(z=0)$ appears at different times for each of the models, namely $t=63, t=58$ and $t=60$ for models 0,1 , and 2 respectively. This can be seen in Fig. 9 (left) where the area of photospheric surface containing flux is charted over time. For model 0 , the first flux will only be seen in the photosphere when the top of $\mathrm{ft} 2$ reaches the top of the convection zone. However, in models 1 and $2 \mathrm{ft} 1$ lies between the top of $\mathrm{ft} 2$ and the photosphere. With $\mathrm{ft} 2$ pushing upwards at $\mathrm{ft} 1$ 's lower edge, the original top of $\mathrm{ft} 1$ can enter the photosphere whilst the top of $\mathrm{ft} 2$ is still several scaleheights below the solar interior photosphere interface. Thus, accounting for the arrival for flux at the photosphere at an earlier time in models 1 and 2 than in model 0 . The increase in the area of the photosphere containing flux occurs at a similar rate for all three of the models since this is related to the rise velocity of flux through the solar interior, initialised by the same buoyancy force for $\mathrm{ft} 2$ in each model.

The transfer of the non-buoyant, magnetic flux into the atmosphere occurs through a magnetic buoyancy instability. The instability is launched most easily by perturbations that bend fieldlines and specifically occurs when the following criterion is satisfied

$-H_{p} \frac{\partial}{\partial z}(\log B)>-\frac{\gamma}{2} \beta \delta+{\tilde{k_{\|}}}^{2}\left(1+\frac{{\tilde{k_{\perp}}}^{2}}{{\tilde{k_{z}}}^{2}}\right)$, 

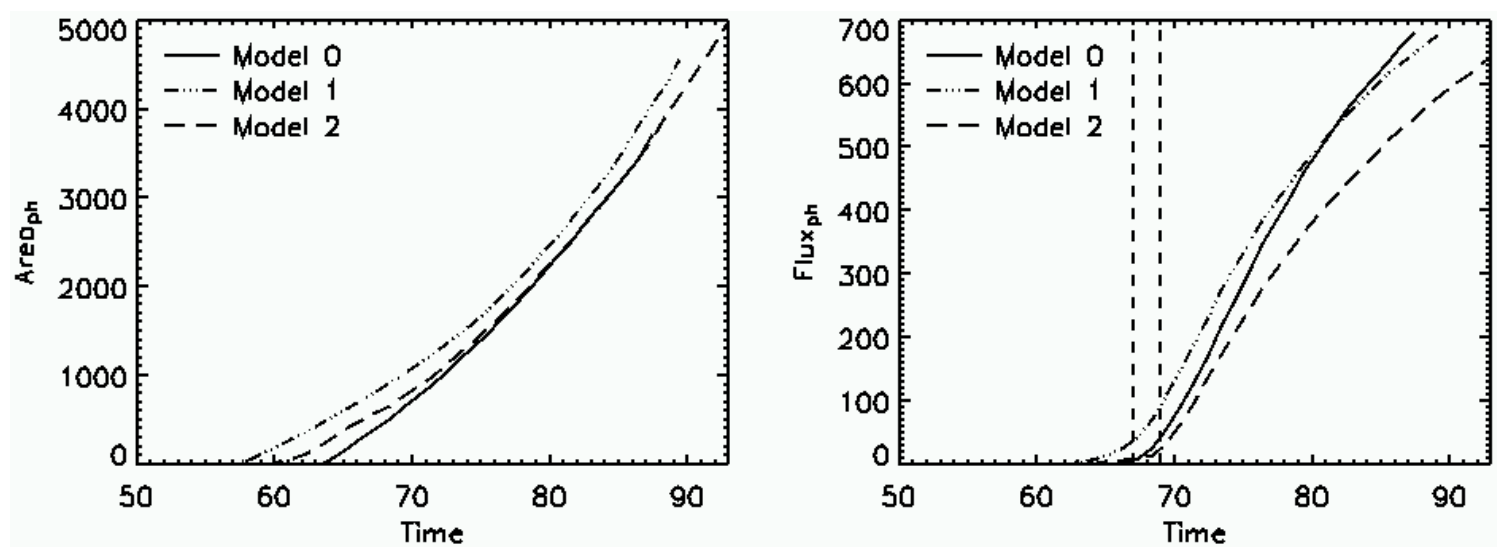

Fig. 9. Increase in the area of the photospheric plane $z=0$ containing flux over time $(l e f t)$ and the change in the vertical unsigned flux measured in the same plane over time (right) for models 0 (solid), 1 (dot-dashed) and 2 (dashed). In the right figure, we indicate the time at which the buoyancy instability is satisfied for models 0 and $1(t=67)$ and model $2(t=69)$ by the first and second lines, respectively.
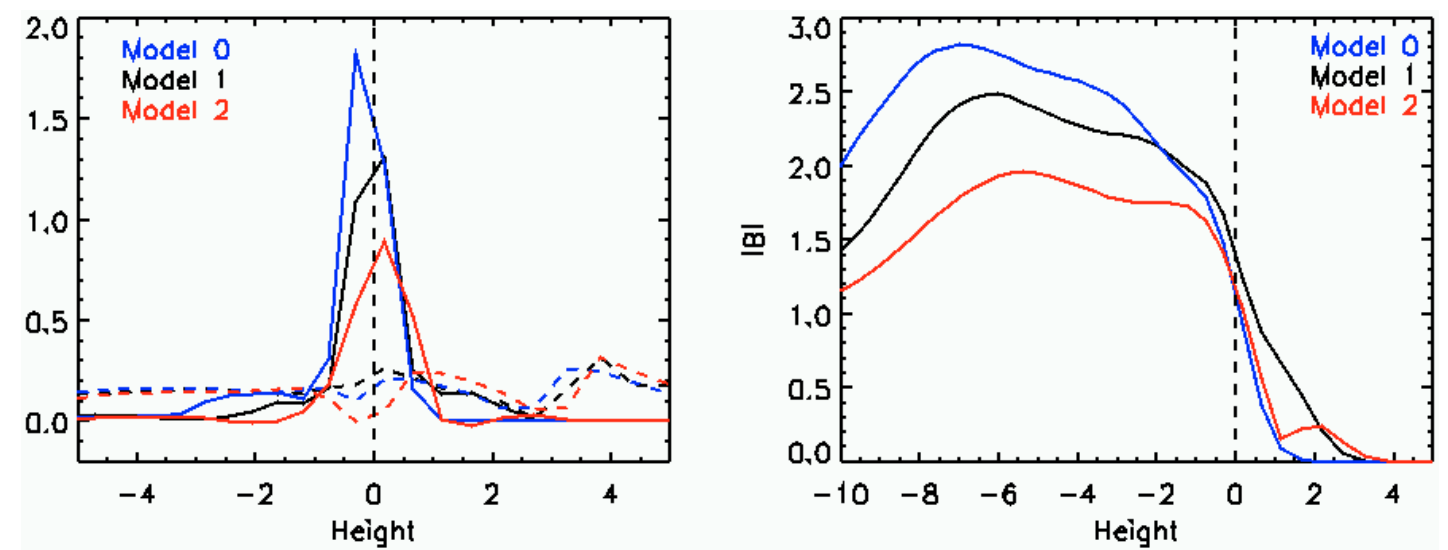

Fig. 10. The magnetic (solid lines) and convective (dashed lines) terms comprising the buoyancy instability (12) (left). The magnitude of the field, $|B|,($ right $)$. The blue, black and red lines represent quantities in models 0,1 and 2 respectively.

(Newcomb 1961; Yu 1965; Thomas \& Nye 1975; Acheson 1979; Archontis et al. 2004). The terms in this criterion are comprised of the photospheric pressure scale-height, $H_{p}$, the ratio of specific heats, $\gamma$, the plasma- $\beta$ and perturbations with wavevector $\boldsymbol{k}$ (where $\tilde{k_{\|}}$and $\tilde{k_{\perp}}$ are the horizontal components parallel and perpendicular to the magnetic field and $\tilde{k}_{z}$ is the vertical component). The superadiabatic excess, $\delta$ is given by $\delta=\nabla-\nabla_{\mathrm{ad}}$, where $\nabla$ is the actual logarithmic temperature gradient in the equilibrium stratification and $\nabla_{\text {ad }}$ is its adiabatic value. Dividing through the lefthand and righthand terms of the inequality by $\beta$, we describe them simply as the magnetic and convective terms respectively.

This inequality has previously been used effectively to demonstrate that tubes, whose magnetic field satisfies (12) when in the photosphere, will emerge (Moreno-Insertis 2006; Murray et al. 2006). Figure 10 (left) shows the magnetic and convective terms comprising the criterion at time $t=67$ for models 0 and 1 and $t=69$ for model 2. At these times the criterion is satisfied for the first time in the photosphere.

The instability is dependent upon the gradient in the field being sufficiently large that it exceeds the convective term. For model 0 , the field at the front of the tube has a naturally steep profile due to the decrease in the field strength with increasing radius, as shown in Fig. 10 (right). The gradient of the profile must still be steepened further but this happens relatively quickly resulting in a difference of only 4 time units between the first field entering the photosphere and the instability occurring. As stated above, when the magnetic field enters the photosphere in models 1 and 2 it is flux that originally belonged to $\mathrm{ft} 1$ and, therefore, it is much weaker than the first field that enters in model 0 , which belongs to $\mathrm{ft} 2$. As in model 0 , the buoyancy instability cannot occur immediately and, in both models 1 and 2, there is a delay of 9 time units until the stronger field of $\mathrm{ft} 2$ enters the photosphere and its profile steepens sufficiently.

Examining Fig. 9 (right), we can see that once the buoyancy instability occurs there is a sharp change in the rate of increase of flux in the photospheric plane for each model. Once emergence starts, stronger tube flux starts to pass through the photosphere on its way to the upper atmosphere, which would have remained trapped below the photosphere had the instability not occurred. Flux advances into the transition region and on towards the corona.

For models 0 and 1 , there is little noticeable difference during the first stages of flux emergence. In contrast, we find an initially complex evolution of the active region for model 2. Figure 11 shows synthesised magnetograms at the photospheric plane for model 2. Overlying the plane, are a selection of green and blue fieldlines traced from $\mathrm{ft} 1$ and $\mathrm{ft} 2$ respectively. When the first flux appears at the plane, it is orientated positive negative when crossing left to right. However, over the course of 11 time units the orientation switches to negative positive from left to right. The reconnection below the surface reduces constraints on the fieldlines and allows them to move. Hence, the segments of field of $\mathrm{ft} 1$, which were originally the uppermost, sink below the 

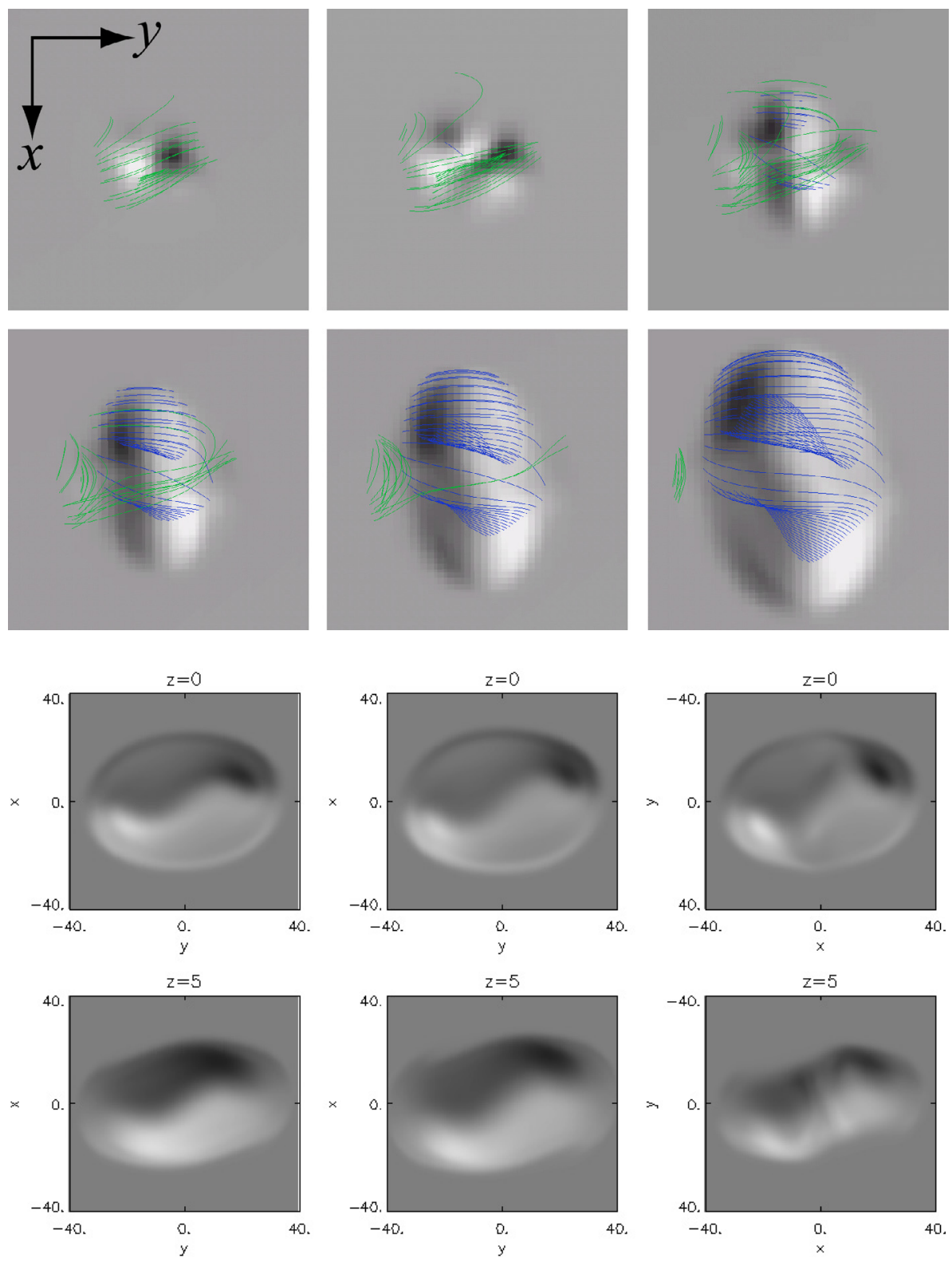
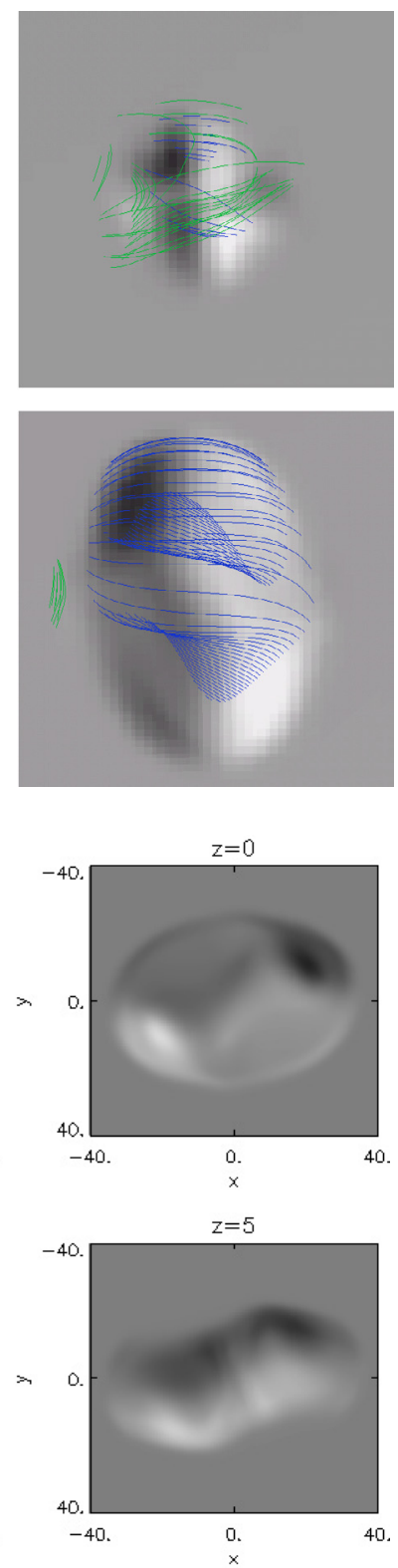

Fig. 11. For model 2, synthesised magnetogram at the horizontal plane $z=0$, with a selection of atmospheric fieldlines traced from the subsurface footpoints of $\mathrm{ft} 1$ (green) and $\mathrm{ft} 2$ (blue). Darker and lighter colourings represent $B_{z}<0$ and $B_{z}>0$ respectively, with the background grey giving the reference for $B_{z}=0$. The images correspond to $t=65,67,69$ (top left, middle, right) and $t=71,73,76$ (bottom left, middle, right).

Fig. 12. Synthesised magnetograms for models $0,1,2$ (left, middle, right), constructed at horizontal planes corresponding to $z=0$ (top row) and $z=5$ (bottom row). Darker and lighter colourings represent $B_{z}<0$ and $B_{z}>0$ respectively, with the background grey giving the reference for $B_{z}=0$. The planes at $z=0$ and $z=5$ have a maximum absolute scaling of $\left|B_{z}\right|=1.0$ and $\left|B_{z}\right|=0.25$ respectively. photosphere revealing the flux of $\mathrm{ft} 2$ as the uppermost flux, labelled E in Fig. 1. From $t=76$ onwards, the evolution of model 2 continues as for the other models.

At $t=80$, the flux pattern at the photospheric plane is the same for all of the models, Fig. 12. Concentrations of positive and negative flux form at opposite edges of the emergence region, where the minimum and maximum line of sight component of the field $\left(B_{z}\right)$ is -0.7 and 0.7 respectively for all three models. As time advances, we find that the peak positive and negative strength in the bipoles increases and at $t=86$ the differences of these values between the three models are only $~ 100$ Gauss. Study of the vertical field at a plane halfway through the photosphere, $z=5$, shows slight differences arise in the case of initially orthogonal tubes. This difference occurs due to a variation in the line along which the buoyancy instability takes place.

At the photospheric plane, we find diverging horizontal velocity flows away from the centre of the flux region, with the strongest flows towards the concentrated bipolar sites. In the three models, the minimum and maximum values of these flows differ by only $\sim 0.1$, equivalent to $680 \mathrm{~m} \mathrm{~s}^{-1}$.

\section{Conclusions}

We have performed three simulations of solar flux emergence. Model 0 consists of a single twisted tube within the solar interior and the other models have two twisted flux tubes below the surface. In models 1 and 2 the tubes are orientated parallel and orthogonal to each other, respectively.

Through the interaction of the two tubes in models 1 and 2, a subsurface magnetic field of greater complexity is created. The new flux system still has tube-like qualities such as a dominant orientation of the axial field and fieldlines twisting about a central structure. Our results are in line with those of previous "reconnecting tubes" studies (Linton et al. 2001; Linton \& Antiochos 2005; Linton 2006). We can, therefore, conclude that the interaction of the tubes is independent of the force driving the tubes together and the plasma- $\beta$ of the environment where the collision event occurs.

In all of our models, the subsurface field emerges into the atmosphere via a magnetic buoyancy instability. At this time reconnection is still occurring in the solar interior in both models 1 and 2. However, this is only evident in the atmospheric 
observations for the case when the two tubes are orthogonal, for which we see a reversal of the bipolar region during flux emergence. To our knowledge, this reversal has not been observed and there are two possible reasons for this. Firstly, the reversal does not occur either because flux tubes in the interior are not orthogonal and, therefore, do not reconnect in this way or because reconnection happens when the tubes are deeper within the solar interior and finishes by the time the emergence occurs. Secondly, the event we see occurs over a period of $4.5 \mathrm{~min}$ but the cadence of MDI magnetograms is $5 \mathrm{~min}$ so the event could pass completely unrecorded. With the launch of Hinode, we hope to be able to resolve the issue of whether bipolar reversal of emerging flux occurs since SOT will produce data with a cadence of $\sim 45 \mathrm{~s}$.

Our synthesised magnetograms, atmospheric fieldline traces and measurements of velocity reveal little difference. This is because emergence in the later stages is dominated by the field of the stronger tube, $\mathrm{ft} 2$. We, therefore, conclude that from these measurements alone it is not possible to determine whether the subsurface field is constructed from one or two flux tubes. A study by Linton \& Priest (2003) investigated the interaction of two orthogonal, untwisted flux tubes when driven together. The tubes flattened into flux sheets and reconnected to form a single twisted flux tube. This suggests that interacting flux sheets would also show no difference during emergence.

At the experiment initialisation stage, we chose the lower tube to be much stronger than the upper tube since previous studies have shown that a flux tube's field strength will decrease as it rises (Archontis et al. 2004; Murray et al. 2006). Although the field strength of the lower tube decreses during its rise, it is still much stronger than that of the upper tube upon contact, as discussed in Sect. 3.2. Thus, the atmospheric results are dominated by the stronger lower tube. We believe that varying the ratio of the tubes' field strengths when they first come into contact will produce few visible atmospheric differences in the case of two parallel tubes but will have greater impact when simulating two orthogonal tubes.

If the orthogonal tubes are of equal strength when they first come into contact, we expect their axes to reconnect with each other, following the findings of Linton et al. (2001). Whether the two tubes would then be able to perform a complete slignshot action prior to emergence is hard to say. This is a highly nongeneric case but, if the tubes were to remain entwined during emergence, more complex photospheric patterns would surely be observed. If the lower tube is weaker than the upper tube upon contact, then the active region would presumably reflect the orientation of $\mathrm{ft} 1$ rather than $\mathrm{ft} 2$. The results would again be similar to those of models 0 and 1 , without the need to rotate the simulation domain in order to achieve similar orientation of the active region. However, these effects are speculative and the necessary investigations have been left for a later date.

From our results we believe that, when studying and investigating generic flux emergence from an atmospheric viewpoint, it is acceptable to simplify simulations and use a single twisted flux tube as the subsurface source of the flux since the atmospheric field gives no indication of the structure of the subsurface field.

Acknowledgements. Michelle Murray acknowledges financial assistance from the Particle Physics and Astronomy Research Council (PPARC). The computational analysis for this paper was carried out on the joint SRIF and PPARC funded UKMHD linux cluster, Copson, in St. Andrews (Scotland, UK). The authors would like to thank the referee for useful suggestions.

\section{References}

Abbett, W. P., Fisher, G. H., Fan, Y., \& Bercik, D. J. 2004, ApJ, 612, 557 Acheson, D. J. 1979, Sol. Phys., 62, 23

Archontis, V., Moreno-Insertis, F., Galsgaard, K., Hood, A., \& O’Shea, E. 2004, A\&A, 426, 1047

Archontis, V., Moreno-Insertis, F., Galsgaard, K., \& Hood, A. W. 2005, ApJ, 635,1299

Dahlburg, R. B., Antiochos, S. K., \& Norton, D. 1997, Phys. Rev. E, 56, 2094

Dorch, S. B. F. 2006, A\&A, 461, 325

Dorch, S. B. F., \& Nordlund, A. 1998, A\&A, 338, 329

Fan, Y. 2001, ApJ, 554, L111

Fan, Y., Zweibel, E. G., \& Lantz, S. R. 1998, ApJ, 493, 480

Fan, Y., Abbett, W. P., \& Fisher, G. H. 2003, ApJ, 582, 1206

Galsgaard, K., Moreno-Insertis, F., Archontis, V., \& Hood, A. 2005, ApJ, 618, L153

Glackin, D. L. 1975, Sol. Phys., 43, 317

Kusano, K., Moriyama, K., \& Miyoshi, T. 1998, Phys. Plasmas, 5, 2582

Linton, M. G. 2006, J. Geophys. Res. (Space Phys.), 111, 12

Linton, M. G., \& Antiochos, S. K. 2005, ApJ, 625, 506

Linton, M. G., \& Priest, E. R. 2003, ApJ, 595, 1259

Linton, M. G., Dahlburg, R. B., \& Antiochos, S. K. 2001, ApJ, 553, 905

Lites, B. W., Low, B. C., Martinez Pillet, V., et al. 1995, ApJ, 446, 877

Matsumoto, R., \& Shibata, K. 1992, PASJ, 44, 167

Moreno-Insertis, F. 2006, in IAU Symp., ed. V. Bothmer, \& A. A. Hady, 33

Moreno-Insertis, F., \& Emonet, T. 1996, ApJ, 472, L53

Murray, M. J., Hood, A. W., Moreno-Insertis, F., Galsgaard, K., \& Archontis, V. 2006, A\&A, 460, 909

Newcomb, W. A. 1961, Phys. Fluids, 4, 391

Nordlund, A., \& Galsgaard, K. 1997, A 3D MHD Code for Parallel Computers,

Tech. rep., Astronomical Observatory, Copenhagen University

Parker, E. N. 1955, ApJ, 121, 491

Parker, E. N. 1979, ApJ, 230, 905

Parnell, C. E., \& Galsgaard, K. 2004, A\&A, 428, 595

Strous, L. H., \& Zwaan, C. 1999, ApJ, 527, 435

Thomas, J. H., \& Nye, A. H. 1975, Phys. Fluids, 18, 490

Yu, C. P. 1965, Phys. Fluids, 8, 650 